\title{
Entraînement de particules par interactions hydrodynamiques dans un champ de force avec écoulement de cisaillement
}

\author{
François Feuillebois ${ }^{\mathrm{a}}$ \\ Laboratoire PMMH, UMR 7636 CNRS, ESPCI, 10 rue Vauquelin, 75231 Paris Cedex 05, France
}

Reçu le 8 octobre 2003, accepté le 11 février 2004

\begin{abstract}
Résumé - Des particules soumises à un champ de force peuvent entraîner d'autres particules non soumises à ce champ par interactions hydrodynamiques. On étudie un processus continu dans lequel les deux types de particules sont transportées par un écoulement de cisaillement perpendiculaire au champ de force. Cet entraînement est modélisé par une étude statistique de la suspension en écoulement de Stokes. Le champ de forces et l'écoulement modifient la probabilité de paire de particules de types différents. On montre que si le cisaillement est trop important, il apparaît des doublets permanents, ce qui augmente l'entraînement. Sinon, cette probabilité est anisotrope, mais est cependant proche de celle calculée pour un champ de force seul, probabilité qui est isotrope. On présente alors un modèle d'entraînement basé sur les vitesses moyennes des deux espèces de particules. Ce problème s'applique en particulier à la technique de séparation dite par cellule SPLITT en chimie analytique. Dans cette technique, des particules marquées soumises à un champ sont séparées mais entraînent les particules non marquées. Le modèle est appliqué à l'optimisation du dimensionnement de la cellule SPLITT.
\end{abstract}

Mots clés : Suspension / statistique / sédimentation / cisaillement / séparation / SPLITT

Abstract - Entrainment of particles by hydrodynamic interactions in a force field and with shear flow. Particles submitted to a force field may entrain by hydrodynamic interactions other particles which are not submitted to that field. A continuous process is considered in which both types of particles are transported by a shear flow that is perpendicular to the force field. This entrainment process is modeled by a statistical analysis of the suspension in Stokes flow. The force field and flow modify the pair probability of particles of different types. It is shown that if the shear rate is too large, permanent doublets appear, which enhances entrainment. Otherwise, this probability is anisotropic, yet close to the probability calculated for the only effect of the force field, that one being isotropic. A model is presented for the average velocities of the two species of particles. This problem applies in particular to the so called SPLITT cell separation technique in analytical chemistry. In this technique, tagged particles are separated but entrain the nontagged ones. The model is applied to optimize the design of dimensions of the SPLITT cell.

Key words: Suspension / statistics / sedimentation / shear / separation / SPLITT

\section{Introduction}

Considérons un mélange de deux types de particules dans un fluide visqueux, par exemple des particules «pesantes $»$ (plus lourdes que le fluide) et des particules « non pesantes » (de même masse volumique que le fluide). Les particules pesantes sédimentent et, ce faisant, créent dans leur voisinage un écoulement qui influe sur le mouvement des particules non pesantes. Ainsi des particules non pesantes peuvent être entraînées vers le

a Auteur correspondant :

Francois.Feuillebois@espci.fr bas. Ce problème d'entraînement collectif de particules par interactions hydrodynamiques entre dans le cadre de la sédimentation d'une suspension polydispersée, qui a été traitée théoriquement de façon statistique pour des sphères de même taille et de poids différents [1] puis pour des sphères de tailles et poids différents $[2,3]$. Des interactions hydrodynamiques collectives ont aussi été étudiées pour des particules sans poids en écoulement de cisaillement [4]. Là, l'objectif était le calcul de la viscosité moyenne d'une suspension. Il est clair que dans différents phénomènes naturels et procédés industriels, l'entraînement hydrodynamique de particules dans le champ de gravité, ou plus généralement dans un champ 


\section{Nomenclature}

\begin{tabular}{|ll|}
\hline$a$ & rayon d'une particule sphérique \\
$c$ & fraction volumique en particules \\
$h$ & distance entre les parois \\
$K$ & nombre sans dimension défini par (6) \\
$n$ & nombre de particules d'indice 1 par unité de volume \\
$P$ & probabilité de paires \\
$p$ & probabilité de paires réduite \\
$R$ & distance du centre d'une particule test non pesante (ou non marquée) \\
& au centre d'une particule pesante (ou marquée) \\
$S$ & coefficient de couplage entre vitesse moyenne et fraction volumique \\
$t$ & temps \\
$\mathbf{v}$ & vitesse du centre d'une particule \\
$\langle\mathbf{v}\rangle$ & vitesse moyenne du centre d'une particule \\
$\mathbf{V}$ & vitesse du centre d'une particule test non pesante (non marquée) \\
& par rapport au centre d'une particule pesante (marquée) \\
$x$ & coordonnée dans le sens de l'écoulement \\
$X$ & $x$ sous forme adimensionnelle \\
$\mathbf{x}$ & position du centre d'une particule \\
$\mathbf{X}$ & vecteur joignant le centre d'une particule test non pesante (non marquée) \\
$z$ & au centre d'une particule pesante (marquée) \\
$Z$ & coordonnée perpendiculaire à l'écoulement et perpendiculaire aux parois \\
Indice 0 & indique une particule non pesante (ou non marquée) \\
Indice 1 & indique une particule pesante (ou marquée) \\
$\kappa$ & taux de cisaillement local de l'écoulement ambiant \\
\hline
\end{tabular}

de force, peut se combiner à l'entraînement dans un écoulement porteur.

On pourra citer le mouvement de sédiments dans des rivières, l'entraînement de particules par flottation (les particules qui entraînent étant alors des bulles), le mouvement de particules dans différents réacteurs, etc. Ici, on considère comme application type la technique de séparation en cellule SPLITT (« Split-flow thin fractionation ») utilisée en chimie analytique. Cette technique permet d'extraire de façon continue certains types de petites particules en suspension dans un fluide porteur [5]. Schématiquement (Fig. 1), la technique consiste à injecter un mélange de particules dans une des deux branches d'entrée de la cellule SPLITT, à dévier au moyen d'un champ des particules choisies marquées sensibles à ce champ, enfin à récupérer les particules voulues dans une des deux branches de la sortie. Pratiquement, on peut régler les débits relatifs dans les deux branches d'entrée, ce qui revient à régler la position de la barrière séparant les deux branches d'entrée; et de même pour les deux branches de sortie. L'entraînement hydrodynamique est un effet parasite pour ce procédé : les interactions hydrodynamiques entre particules marquées et particules non marquées se traduisent par une perte de résolution de la séparation. En effet, des particules marquées peuvent entraîner des particules non marquées vers la sortie opposée (Fig. 1).

Cet article concerne la modélisation théorique de l'entraînement par interactions hydrodynamiques collectives. On considère deux types de particules dans un écoulement

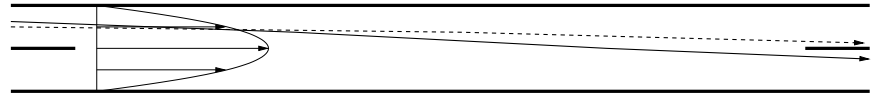

Fig. 1. Schéma de la cellule SPLITT. Une suspension de particules est injectée dans la branche en haut à gauche et du fluide pur dans la partie basse. Une particule marquée (trajectoire en trait plein) se déplace latéralement et peut être séparée en sortant en bas à droite. Une particule non marquée (trajectoire en trait pointillé) ne migre pas et doit en principe sortir en haut à droite; mais elle risque d'être entraînée par les particules marquées et peut alors sortir avec elles.

de Poiseuille entre deux plaques parallèles. Certaines particules marquées sont soumises à un champ de force perpendiculaire à l'écoulement et les autres particules non marquées sont insensibles à ce champ. Pour fixer les idées, nous considérerons des particules de l'ordre de la dizaine de $\mu \mathrm{m}$ dans de l'eau, conditions utilisées dans la cellule SPLITT. L'écoulement autour des particules est à petit nombre de Reynolds et l'on utilisera l'approximation de Stokes. En outre, le nombre de Péclet est grand et le mouvement brownien est négligeable. Pour simplifier le problème on supposera les particules solides et sphériques, de même rayon $a$.

Nous allons d'abord présenter dans la section 2 des trajectoires de deux particules (une de chaque type) en interaction hydrodynamique, mettant en évidence l'effet d'entraînement. Puis le phénomène à l'échelle de la suspension sera modélisé ici par une étude statistique. 
La première partie consistera à calculer la probabilité d'avoir une particule marquée au voisinage d'une particule non marquée, section 3. Puis nous écrirons un modèle pour le comportement global de la suspension, section 4 . Comme application, nous en déduirons alors un dimensionnement de la cellule SPLITT propre à supprimer ce phénomène d'entraînement, section 5 .

\section{Trajectoires de deux particules en interaction}

L'effet essentiel étudié consiste en l'entraînement d'une particule non marquée par une particule marquée. Ce problème est analogue à celui d'une particule pesante en sédimentation entraînant une particule non pesante, mais ici les deux particules sont en outre entraînées par l'écoulement de Poiseuille.

La masse volumique des particules étant voisine de celle du fluide, l'inertie des particules est négligeable puisque celle du fluide est négligée. On utilise alors les résultats classiques pour les mobilités de particules en interaction, en considérant que celles-ci tournent librement (aucun couple extérieur n'est ici appliqué aux particules). Les équations de Stokes étant linéaires, on peut superposer les mouvements relatifs dûs à la sédimentation (d'après [6]), à un écoulement de cisaillement local autour des particules (d'après [7]) et à un écoulement parabolique. Nous n'avons pas trouvé de résultat pour ce dernier cas, mais une approximation peut être facilement calculée en utilisant le premier terme de la méthode des réflexions : l'écoulement perturbé produit par une sphère en écoulement parabolique est calculé d'après [8], puis la contribution de cet écoulement perturbé à la vitesse de l'autre sphère est obtenue grâce à la formule de Fàxen (cf. par exemple [9]). La quantité ainsi calculée est d'ordre $(a / h)^{2}$, où $h$ est la distance entre les parois. Nous avons gardé ce terme dans le calcul de trajectoires, mais il reste petit, les particules étant petites devant l'intervalle entre les parois. Notons que les interactions entre une particule et les deux parois, la particule étant éloignée de la paroi, donneraient un terme d'ordre $(a / h)^{3}[10]$, terme que nous négligerons ici. En outre, les effets liés aux particules proches des parois sont secondaires ici.

Lorsque les particules sont proches, les forces de lubrification s'appliquent. Nous avons utilisé ici l'ensemble des formules classiques $[6,11]$. Comme ces forces deviennent grandes lorsque l'intervalle entre particules devient petit, le système des équations du mouvement est raide. Aussi, nous avons utilisé pour l'intégrer une méthode de type prédicteur-correcteur [12].

Un exemple typique de deux trajectoires couplées est présenté figure 2. L'épaisseur du canal est ici de $100 a$. La vitesse au centre du canal vaut 5 fois la vitesse de « sédimentation » de la particule « pesante (marquée). La sphère non marquée (notée « 0 *) est placée initialement en $(0,99 ; 0 ; 69,52)$ et la sphère marquée (notée « $1 »)$ est en $(0 ; 0 ; 71,26)$. Ces positions ont été choisies de façon que les sphères passent près l'une de l'autre. On voit sur

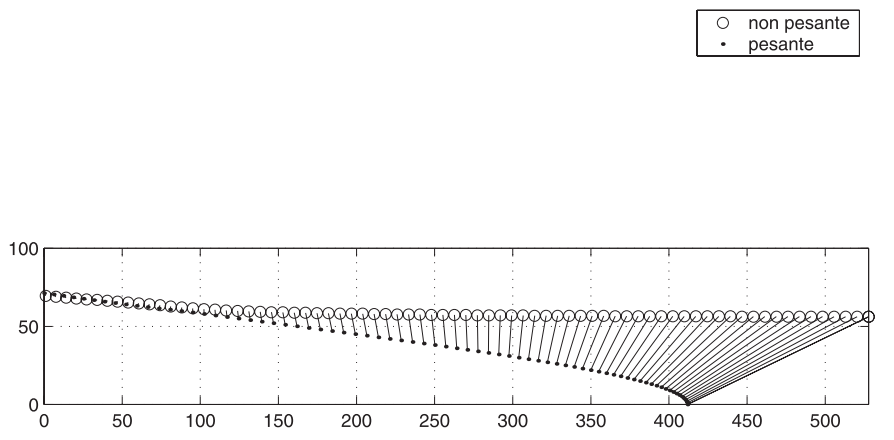

Fig. 2. Trajectoires de deux particules en interaction en écoulement de Poiseuille. Les coordonnées adimensionnelles sont rapportées au rayon d'une sphère. Les positions des deux particules à un instant donné sont jointes par un segment de droite.

la figure 2 que la particule non marquée est bien entraînée en travers de l'écoulement par la particule marquée.

\section{Probabilité de paires}

Compte-tenu du grand nombre de particules qui interagissent entre elles par l'intermédiaire du fluide, une étude statistique est appropriée. La fraction volumique de la suspension étant petite devant l'unité, il suffit d'étudier les interactions de paires de particules, comme dans les cas classiques de calculs de la vitesse moyenne de sédimentation $[3,13]$ et de la viscosité moyenne d'un écoulement [4]. Nous commençons par étudier la probabilité de trouver une particule marquée au voisinage d'une particule non marquée donnée, compte-tenu du mouvement relatif de ces deux types de particules. Puis, sur la base de cette probabilité, nous estimerons (Sect. 4) les vitesses moyennes d'entraînement des particules non marquées.

La probabilité d'observer une particule 1 centrée en $\mathbf{x}_{1}=\mathbf{x}_{0}+\mathbf{X}$ lorsqu'une particule test 0 est centrée en $\mathbf{x}_{0}$ s'écrit :

$$
P\left(\mathbf{x}_{0}+\mathbf{X} \mid \mathbf{x}_{0}\right)= \begin{cases}0 & \text { si } R<2 a \\ n p(\mathbf{X}) & \text { si } R>2 a\end{cases}
$$

où $n$ est le nombre de particules 1 par unité de volume et $R=|\mathbf{X}|$. La probabilité est nulle si la distance $R$ entre centres est inférieure à deux rayons, c'est-à-dire que les particules 0 et 1 ne s'interpénètrent pas. La probabilité réduite $p(\mathbf{X})$ est solution d'une équation de FokkerPlanck :

$$
\frac{\partial p(\mathbf{X})}{\partial t}+\nabla_{\mathbf{X}} \cdot\left[p(\mathbf{X}) \mathbf{V}\left(\mathbf{x}_{0}, \mathbf{X}\right)\right]=0
$$

où $\mathbf{V}\left(\mathbf{x}_{0}, \mathbf{X}\right)$ est la vitesse relative du centre de la sphère 1 par rapport au centre de la sphère 0 . Cette équation peut s'obtenir en considérant que c'est l'équation de continuité du nuage des centres des particules marquées, analogue à un gaz de masse volumique $p(\mathbf{X})$ et de vitesse $\mathbf{V}$ dans le repère de $\mathbf{x}_{0}$. 
On adjoint à l'équation (2) l'hypothèse que des sphères très éloignées l'une de l'autre sont statistiquement indépendantes, soit

$$
p(\mathbf{X}) \rightarrow 1 \text { pour }|\mathbf{X}| \rightarrow \infty
$$

Notons que l'équation (2) peut s'écrire sous forme lagrangienne :

$$
\frac{\mathrm{d} \log p(\mathbf{X})}{\mathrm{d} t}=-\nabla_{\mathbf{X}} \cdot \mathbf{V}\left(\mathbf{x}_{0}, \mathbf{X}\right)
$$

où $\mathrm{d} / \mathrm{d} t$ désigne la dérivée particulaire en suivant la particule 1 dans son mouvement par rapport à la particule 0 . La divergence du membre de droite (ou compressibilité) se calcule analytiquement connaissant l'expression de la vitesse relative. Cette équation doit être couplée à celle du mouvement relatif

$$
\frac{\mathrm{d} \mathbf{X}}{\mathrm{d} t}=\mathbf{V}\left(\mathbf{x}_{0}, \mathbf{X}\right)
$$

On intègre alors numériquement le système différentiel (4)(5) avec la condition (3) en fonction du temps. Comme pour les trajectoires, il faut utiliser une méthode de type prédicteur-correcteur.

Dans le cas d'un cisaillement pur, Batchelor \& Green [4] ont indiqué que les trajectoires relatives peuvent être fermées; la condition à l'infini (3) pour $p$ n'est pas applicable. Ceci peut intervenir aussi ici si l'effet de cisaillement est trop important par rapport à l'effet de sédimentation. Si $V_{1}$ est la vitesse de migration d'une particule marquée sous l'action du champ et $\kappa$ le taux de cisaillement local, un nombre caractéristique sans dimension qui apparaît est

$$
K=\kappa a / V_{1}
$$

Pour une valeur de $K$ donnée assez grande, il existe des doublets permanents lorsque les particules sont assez proches. Soit un système de coordonnées cartésiennes $(X, Y, Z)$ adimensionnelles par rapport au rayon et ayant pour origine le centre de la particule test non marquée. Soit $X, Y$ le plan de l'écoulement de cisaillement avec $X$ suivant la direction de l'écoulement. $Z$ est dans la direction du champ de forces mais dans le sens opposé. Considérons le centre d'une particule marquée en $Y=Z=0$ et $X<0$ donnée. Le champ de forces tire la particule marquée vers $Z<0$ et l'écoulement de cisaillement tend à la faire contourner l'autre sphère en direction de $Z>0$. En écrivant l'équilibre de ces forces, on trouve une valeur limite de $K$ pour ce point $(X, Y, Z)$. Ainsi par exemple si $X=-3$ (soit un intervalle d'un rayon entre les sphères), on trouve une valeur limite $K_{\lim }=21,8334$. Les trajectoires relatives de sphères plus proches, c'està-dire passant par $|X|<3, Y=Z=0$, sont fermées comme le montre par exemple la figure 3 pour laquelle $X=-2,9$. Alors que les trajectoires de sphères plus éloignées sont ouvertes. L'intervalle de $X$ donnant des trajectoires fermées diminue lorsque $K$ diminue et l'on trouve que pour $K<K_{\lim , \min }=0,9901$ les trajectoires sont toujours ouvertes.

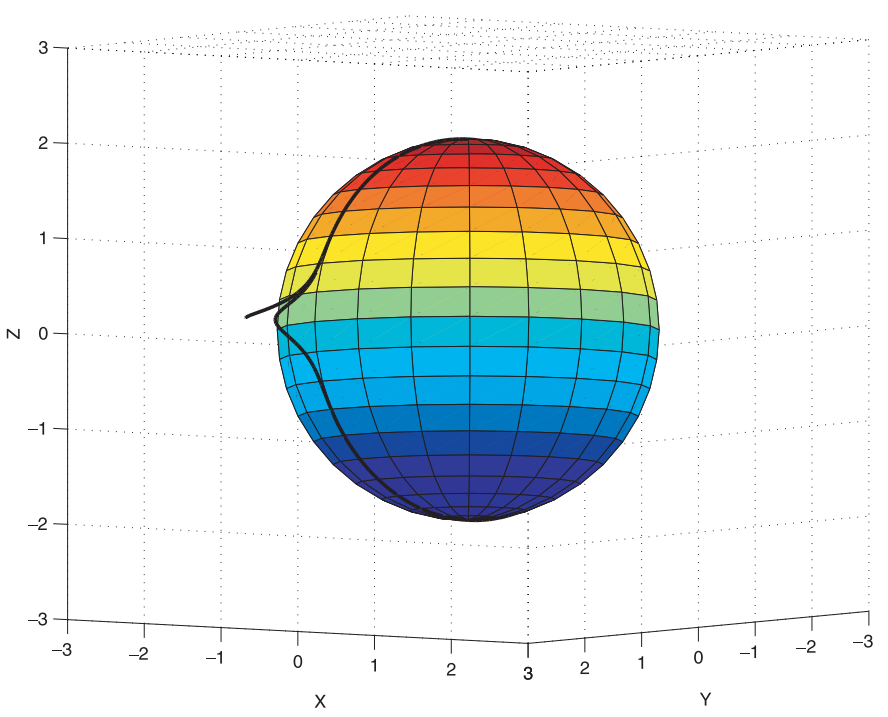

Fig. 3. Trajectoire relative (en trait plein) du centre d'une sphère marquée par rapport au centre d'une sphère non marquée, pour $K=21,8334$. Les coordonnées adimensionnelles sont rapportées au rayon d'une sphère. La sphère de rayon 2 est la sphère de contact (lieu des points pour lesquels le centre de la sphère marquée est en contact avec le centre de la sphère non marquée). On voit que la trajectoire passant par $(X=-2,9 ; Y=Z=0)$ est fermée : il apparaît ici un doublet permanent qui favorise l'entraînement.

Si $a$ et $V_{1}$ sont donnés et si l'on veut que toutes les trajectoires soient ouvertes, on a donc une contrainte sur le débit volumique maximum, en prenant pour $\kappa$ le taux de cisaillement maximum de l'écoulement de Poiseuille :

$$
Q<\frac{1}{6} \frac{h^{2}}{a} V_{1} K_{\text {lim,min }} \simeq 0,165 \frac{h^{2}}{a} V_{1}
$$

Dans le cas où les trajectoires relatives sont toujours ouvertes, les résultats obtenus pour $p$ indiquent que la probabilité est anisotrope, alors qu'elle est isotrope dans les cas particuliers du cisaillement seul et de la sédimentation seule. Néanmoins, l'anisotropie reste faible et les valeurs obtenues pour la probabilité de paires sont proches (à $10 \%$ près) de celles obtenues pour la sédimentation seule [1,3]. Pour simplifier, on pourra donc adopter les valeurs numériques calculées dans $[1,3]$.

\section{Comportement statistique du mélange de particules}

Dans ces conditions, on utilisera les coefficients de sédimentation calculés avec la probabilité de paires de la sédimentation pure. La vitesse moyenne des particules non marquées créée par le mouvement des particules marquées est alors :

$$
\left\langle v_{0}\right\rangle=S_{01} c_{1} V_{1} \quad \text { avec } \quad S_{01} \simeq 0,1
$$

où $c_{1}$ est la fraction volumique des particules marquées et $V_{1}$ la vitesse de migration des particules 1 sous l'action du 
champ. Compte-tenu de l'approximation faite sur $p$ (soit à $10 \%$ près), on ne retient ici qu'une décimale pour le coefficient de couplage $S_{01}$.

Notons que les particules non marquées doivent freiner les particules marquées. En outre, les particules marquées interagissent aussi entre elles et donc se freinent mutuellement. La vitesse moyenne des particules marquées est alors, en ne considérant que les interactions de paires :

$$
\left\langle v_{1}\right\rangle=\left(S_{10} c_{0}+S_{11} c_{1}\right) V_{1}
$$

D'après [3], pour le cas de la sédimentation, l'effet de particules non pesantes sur la vitesse moyenne de particules pesantes revient à remplacer la viscosité $\mu$ du fluide par une viscosité équivalente $\langle\mu\rangle=\mu\left(1+5 / 2 c_{0}\right)$ (formule d'Einstein), où $c_{0}$ est la fraction volumique des particules non pesantes. Alors, au premier ordre en fraction volumique, $S_{10}=-2,5$. Toujours dans le cas de la sédimentation, il existe pour des particules non browniennes une singularité pour une suspension polydisperse au voisinage de la monodispersité [3] ; pour des particules soumises à la même force extérieure et dans la limite d'un rapport des rayons tendant vers l'unité, $S_{11} \simeq-5,6$.

Pour un champ de force couplé à un écoulement, il faudrait refaire les calculs en considérant en outre le cas polydispersé. On peut penser que les coefficients $S_{10}$ et $S_{11}$ seront proches de celui de la sédimentation, comme pour le coefficient de couplage $S_{01}$. Mais l'effet principal est celui d'entraînement et ces coefficients n'apportent que des corrections.

\section{Dimensionnement de la cellule SPLITT}

Comme indiqué dans l'introduction, la cellule SPLITT est un cas typique d'application de ce problème d'entraînement de particules non marquées par des particules marquées. Nous allons montrer que les dimensions de la cellule SPLITT peuvent être ajustées de façon à supprimer en principe le phénomène d'entraînement, phénomène ici parasite comme on l'a vu plus haut. On suppose pour simplifier que l'écoulement dans la cellule SPLITT est un écoulement de Poiseuille. En effet, l'épaisseur de la cellule étant inférieure au $\mathrm{mm}$ et la longueur de l'ordre de quelques dizaines de $\mathrm{cm}$, les zones d'entrée et de sortie (Fig. 1) sont négligeables. Pour simplifier, on néglige aussi le freinage des particules marquées par les particules non marquées et les autres particules marquées et, dans le sens de l'écoulement, on suppose que les particules se déplacent à la vitesse de l'écoulement de Poiseuille. Soit $h$ l'épaisseur de la cellule et $z$ la coordonnée suivant l'épaisseur. Les particules sont injectées à l'entrée entre $z=h_{\mathrm{i}}$ et $z=h$ et du fluide clair est injecté entre $z=0$ et $z=h_{\mathrm{i}}$. Les particules 1 sont soumises à un champ dirigé vers $z=0$. La sortie est séparée en deux en $z=h_{\mathrm{s}}$. On veut faire sortir les particules 1 entre $z=0$ et $z=h_{\mathrm{s}}$ et les particules 0 entre $z=h_{\mathrm{s}}$ et $z=h$. On veut également que des particules 1 n'arrivent pas à la paroi $z=0$ avant la sortie, auquel cas certaines de ces particules ne pourraient pas être récupérées.

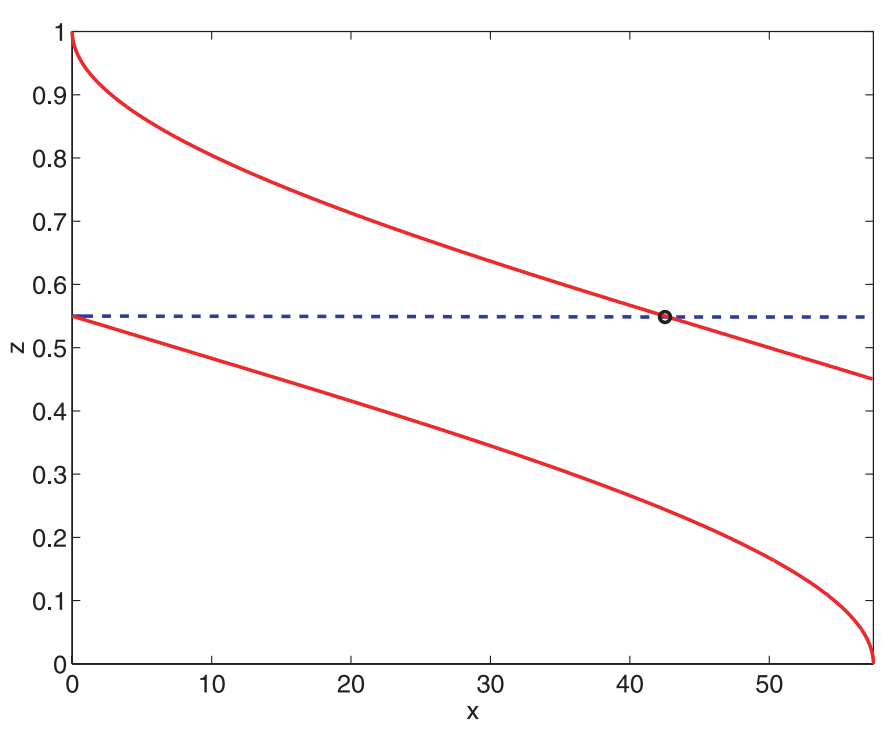

Fig. 4. Trajectoires extrêmes des particules marquées (en trait plein) et des particules non marquées (en trait pointillé; la trajectoire supérieure ne se distingue pas de $Z=1$ ). Les coordonnées sans dimension sont rapportées à l'épaisseur de la cellule.

On trouve que pour ne pas capter de particules non marquées, la sortie $h_{\mathrm{s}}$ devra être placée en

$$
h_{\mathrm{s}}<z_{\mathrm{s}}=h_{\mathrm{i}}-\frac{1}{6} c_{1} S_{01}\left(h-h_{\mathrm{i}}\right)\left(h+2 h_{\mathrm{i}}\right) / h_{\mathrm{i}}
$$

et à une distance vers l'aval

$$
x>x_{\mathrm{s}}=\left(\bar{u} / V_{1}\right) h\left[1-3\left(h_{\mathrm{i}} / h\right)^{2}+2\left(h_{\mathrm{i}} / h\right)^{3}\right],
$$

où $\bar{u}$ est la vitesse moyenne de l'écoulement de Poiseuille. À cette distance, les particules marquées ne doivent pas avoir touché la paroi $z=0$; ceci est réalisé si $h_{\mathrm{i}}>h / 2$. Ainsi, on voit que la tendance naturelle à séparer le canal en deux parties égales à l'entrée n'est pas la meilleure solution. On a tracé figure 4 un exemple de cellule où la séparation à l'entrée est $h_{\mathrm{i}} / h=0,55$. Les coordonnées $X$ suivant l'écoulement et $Z$ en travers de l'écoulement sont normalisées par l'épaisseur $h$ de la cellule. La fraction volumique en particules marquées est $c_{1}=5 \%$. Le rapport des vitesses est $\bar{u} / V_{1}=100$. Le point important de l'écoulement est celui où la trajectoire des particules non marquées partant de $Z=h_{\mathrm{i}} / h$ croise la trajectoire des particules marquées partant de $Z=1$. Ce point, qui apparaît plus nettement sur l'agrandissement de la figure 5 , a pour coordonnées $X_{\mathrm{s}}=x_{\mathrm{s}} / h$ et $Z_{\mathrm{s}}=z_{\mathrm{s}} / h$; il permet de dimensionner la cellule comme indiqué ci-dessus. Dans le cas de la figure 4, on voit que l'on a une fourchette de valeurs pour la longueur de la cellule; on peut prendre par exemple $X=55$ (longueur égale à 55 fois l'épaisseur), avec une séparation de sortie à $Z=h_{\mathrm{s}} / h=0,5$.

Il existe divers types de champs utilisés pour déplacer les particules marquées. On utilise en particulier un gradient de champ magnétique. L'application en vue est la séparation de cellules sanguines souches. Il existe en effet des techniques biologiques permettant d'attacher des 


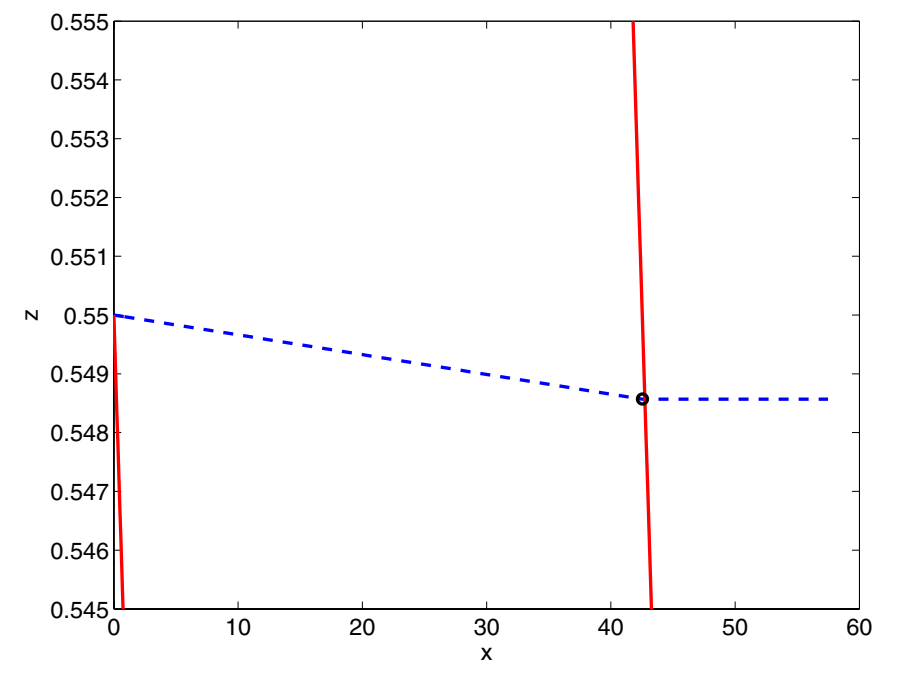

Fig. 5. Agrandissement de la partie de la figure 4 proche de la trajectoire inférieure des particules non marquées : ce tracé met en évidence la déviation de ces particules par les particules marquées.

particules magnétiques à ces cellules. Des expériences préliminaires [5] ont été réalisées avec des particules modèles. Un autre effet parasite est alors dû à la gravité. En effet, les particules à séparer et les autres particules ont leurs densités propres sur lesquelles on ne peut pas agir. Si la cellule était horizontale la gravité donnerait des déplacements parasites dans la même direction que le champ magnétique. On place donc la cellule verticalement. Or, des expériences réalisées en orientant l'écoulement vers le haut et vers le bas montrent que les résultats de la séparation sont différents. Des expériences réalisées en microgravité montrent que les résultats sont encore différents [14]. Ceci peut s'interpréter par l'action de forces de portance qui se traduisent par un effet de migration des particules perpendiculairement aux lignes de courant. Il s'agit d'un effet hydrodynamique qui existe même en l'absence de champ magnétique [15]. Cet effet est lié à un couplage entre l'écoulement de cisaillement de l'écoulement et la gravité, lorsque les deux sont dans la même direction. Le modèle précédent permet donc ici de se placer dans une situation où l'on évite un couplage complexe entre deux effets parasites.

\section{Conclusion}

Le mouvement d'entraînement hydrodynamique de particules non marquées par des particules marquées se déplaçant dans un champ de force a été modélisé par une approche statistique. Il est apparu que des doublets permanents se forment si le taux de cisaillement $\kappa$ de l'écoulement ambiant est supérieur à $0,9901 V_{1} / a$. Les doublets formés peuvent alors être quantifiés plus précisément d'après l'étude ci-dessus. Ces doublets permanents favorisent l'entraînement. Dans le cas où il n'existe pas de doublet permanent, la probabilité de paires a été trouvée proche de celle de la sédimentation. On peut alors donner une valeur approchée du coefficient de couplage dans l'équation (7) de la vitesse moyenne d'entraînement. Une étude plus complète devrait prendre en compte la polydispersité pour évaluer plus précisément les différents coefficients de freinage des particules marquées dans l'équation (8).

On a alors, grâce à ce modèle, optimisé les proportions de la cellule SPLITT de façon à supprimer théoriquement les effets d'entraînement hydrodynamique. On a en outre obtenu une limite supérieure du débit de l'écoulement dans la cellule au delà de laquelle il peut exister des doublets permanents, qui sont à éviter.

\section{Références}

[1] F. Feuillebois, Certains problèmes d'écoulements mixtes fluide-particules solides, Thèse d'État, Université de Paris 6, 1980

[2] G.K. Batchelor, Sedimentation in a dilute polydisperse system of interacting spheres, Part 1: general theory, J. Fluid Mech. 119 (1982) 379-408

[3] G.K. Batchelor, C.S. Wen, Sedimentation in a dilute polydisperse system of interacting spheres, Part 2: numerical results, J. Fluid Mech. 124 (1982) 495-528

[4] G.K. Batchelor, J.T. Green, The determination of the bulk stress in a suspension of spherical particles to or$\operatorname{der} c^{2}$, J. Fluid Mech. 56 (1972) 401-427

[5] M. Hoyos, L.R. Moore, K.E. McCloskey, S. Margel, M. Zuberi, J.J. Chalmers, M. Zborowski, Study of magnetic particles pulse-injected into an annular SPLITTlike channel inside a quadrupole magnetic field, J. Chromatography A 903 (2000) 99-116

[6] D.J. Jeffrey, Y. Onishi, Calculation of the resistance and mobility functions for two unequal rigid spheres in lowReynolds-number flow, J. Fluid Mechanics 139 (1984) 261-290

[7] G.K. Batchelor, J.T. Green, The hydrodynamic interaction of two small freely-moving spheres in a linear flow field, J. Fluid Mech. 56 (1972) 375-400

[8] A.T. Chwang, Hydrodynamics of low-Reynolds-number flow, Part 3, Motion of a spheroidal particle in quadratic flows, J. Fluid Mech. 72(1) (1975) 17-34

[9] J. Happel, H. Brenner, Low Reynolds number hydrodynamics, Kluwer Academic Publishers, Dordrecht, Boston, London, 1991

[10] B.P. Ho, L.G. Leal, Inertial migration of rigid spheres in two-dimensional unidirectional flows, J. Fluid Mech. 65 (1974) 365-400

[11] S. Kim, S.J. Karrila, Microhydrodynamics: Principles and Selected Applications, Butterworth-Heineman, 1991

[12] The Mathworks, Function ode15s (to solve stiff ODEs), Technical report, Matlab, version 6.5 release 13, 2002

[13] G.K. Batchelor, Sedimentation in a dilute dispersion of spheres, J. Fluid Mech. 52 (1972) 245-268

[14] M. Hoyos, P. Kurowski, Rôle des forces de portance et diffusion hydrodynamique dans les processus de séparation par la technique de SPLITT, J. Phys. IV France 11 (2001) Pr6-73-Pr6-81

[15] P. Vasseur, R.G. Cox, The lateral migration of a spherical particle in two-dimensional shear flows, J. Fluid Mech. 78 (1976) 385-413 